2. - - Hilbert space is homeomorphic to the countable infinite product of lines, Bull. Amer. Math. Soc. 72 (1966), 515-519.

3. V. L. Klee, Convex bodies and periodic homeomorphism in Hilbert spaces, Trans. Amer. Math. Soc. 74 (1953), 10-43.

4. — A note on topological properties of normed linear spaces, Proc. Amer. Math. Soc. 7 (1956), 673-674.

5. Bor-Luh Lin, Two topological problems concerning infinite-dimensional normed linear spaces, Trans. Amer. Math. Soc. 114 (1965), 156-175.

6. J. R. Retherford and C. W. McArthur, Some remarks on bases in linear topological spaces, Math. Ann. 64 (1966), 38-41.

Louisiana State University

\title{
A SELECTION THEOREM
}

\section{E. MICHAEL ${ }^{1}$}

1. Introduction. The following theorem was proved in $[1$, Footnote 7]. (A function $\phi$ from $X$ to the collection $2^{B}$ of nonempty closed subsets of $B$ is called lower semicontinuous (=l.s.c.) if $\{x \in X: \phi(x) \cap V \neq \varnothing\}$ is open in $X$ whenever $V$ is open in $B$, while $\Gamma_{B} A$ denotes the closed convex hull of $A$ in $B$.)

THEOREM 1.1 [1]. If $X$ is paracompact, if $B$ is a Banach space, and if $\phi: X \rightarrow 2^{B}$ is l.s.c., then there is a continuous $f: X \rightarrow Y$ such that $f(x) \in \Gamma_{B} \phi(x)$ for every $x \in X$.

As was pointed out in $[1$, p. 364], Theorem 1.1 remains true if $B$ is any complete, metrizable locally convex space, but it is generally false if $B$ is not metrizable. We can, however, prove the following generalization of Theorem 1.1.

Theorem 1.2. Let $X$ be paracompact, and $M$ a metrizable subset of a complete $^{2}$ locally convex space E. Let $\phi: X \rightarrow 2^{M}$ be l.s.c. and such that, for some metric on $M$, every $\phi(x)$ is complete. Then there exists a continuous $f: X \rightarrow E$ such that $f(x) \in \Gamma_{E} \phi(x)$ for every $x \in X$.

Theorem 1.2 was proved in [3] under the stronger assumption that $X$ is metrizable. While that was sufficient for the applications in [3], and probably for most other applications, it did not generalize Theorem 1.1, and was therefore never entirely satisfying. In this

Received by the editors May 23, 1966.

1 Supported by NSF Grant 11-5020.

${ }^{2}$ It suffices if $\Gamma_{E} K$ is compact for every compact $K \subset M$. 
paper, some machinery is created in $\$ 2$ which enables us to prove Theorem 1.2 in full generality.

2. Two lemmas. Let $M$ be a metric space with metric $\rho$, and $L$ the linear space of real-valued Lipschitz functions on $X$. As was shown in [4], there is a Banach space $B$ containing $M$ isometrically, and a linear map $f \rightarrow f^{*}$ from $L$ to the dual space of $B$, such that $f^{*} \mid M=f$ for all $f \in L$.

For each $f \in L$, let $s(f)=\{x \in M: f(x) \neq 0\}$ - For each $y \in B$, let $\mathcal{S}(y)=\left\{U \subset M: U\right.$ open, $f^{*}(y)=0$ whenever $f \in L$ and $\left.s(f) \subset U\right\}$, and let $\sigma(y)=M-\cup S(y)$. Clearly $\sigma(y)$ is closed.

The following lemmas are not the sharpest results possible, but they suffice for our purposes.

Lemma 2.1. Suppose that $K \subset M$ is compact, and that $y \in \Gamma_{B} K$. Then

(a) $\sigma(y) \subset K$.

(b) If $f \in L$, and $\sigma(y) \cap s(f)=\varnothing$, then $f^{*}(y)=0$.

(c) $\sigma(y) \neq \varnothing$.

Proof. (a) Let $U=M-K$. Then $f^{*}(y)=0$ whenever $s(f) \subset U$, so $U \in \mathcal{S}(y)$ and hence $\sigma(y) \subset K$.

(b) Since $f=f^{+}-f^{-}$, where $f^{+} \geqq 0$ and $f^{-} \geqq 0$ and both are in $L$, we need only prove (b) for $f \geqq 0$.

Let $A=s(f) \cap K$. Then $A$ is compact and disjoint from $\sigma(y)$, so it can be covered by open $V_{i} \subset X(i=1, \cdots, n)$ such that $\bar{V}_{i} \subset U_{i}$ for some $U_{i} \in s(y)$. Let $g_{i}(x)=\rho\left(x, X-V_{i}\right)$ for all $x \in M$. Then $g_{i} \in L$ and $s\left(g_{i}\right) \subset U_{i}$, so $g_{i}^{*}(y)=0$. Let $g=\sum_{i=1}^{n} g_{i}$. Then $g^{*}(y)=0$. Now $g(x)>0$ when $x \in A$, so there is an $\alpha>0$ such that $0 \leqq f(x) \leqq \alpha g(x)$ for all $x \in A$, and therefore for all $x \in K$ since $f(x)=0$ if $x \in K-A$. Hence $0 \leqq f^{*}(y)$ $\leqq \alpha g^{*}(y)=0$.

(c) Let $h(x)=1$ for all $x \in M$. Then $h \in L$ and $h^{*}(y)=1$, so $\sigma(y)$ $\cap s(h) \neq \varnothing$ by (b). Hence $\sigma(y) \neq \varnothing$, and that completes the proof.

Now let $H=\bigcup\left\{\Gamma_{B} K: K \subset M, K\right.$ compact $\}$, let $\mathcal{K}(M)$ be the set of compact elements of $2^{M}$, and let $\sigma: H \rightarrow \mathcal{K}(M)$ be the map $y \rightarrow \sigma(y)$.

Lemma 2.2. The map $\sigma: H \rightarrow \mathfrak{K}(M)$ is l.s.c.

Proof. Let $V \subset M$ be open, and suppose that $y_{0} \in H$ and $\sigma\left(y_{0}\right)$ $\cap V \neq \phi$. Then there is an $f_{0} \in L$ such that $s\left(f_{0}\right) \subset V$ and $f_{0}{ }^{*}\left(y_{0}\right) \neq 0$. Let $U=\left\{y \in H: f_{0}^{*}(y) \neq 0\right\}$. Then $U$ is a neighborhood $y_{0}$ in $H$, and if $y \in U$, then $\sigma(y) \cap s\left(f_{0}\right) \neq \varnothing$ by Lemma 2.1 (b), so $\sigma(y) \cap V \neq \varnothing$. Hence $\{y \in H: \sigma(y) \cap V \neq \varnothing\}$ is open in $H$, so $\sigma$ is l.s.c.

3. Proof of Theorem 1.2. First, apply [2, Theorem 1.1] to pick a 
1.s.c. map $\psi: X \rightarrow 2^{M}$ such that, for all $x \in X$, we have $\psi(x) \subset \varnothing(x)$ and $\psi(x)$ is compact.

Let $B \supset H \supset M$ be as in $\S 2$, and apply Theorem 1.1 to pick a continuous $g: X \rightarrow B$ such that $g(x) \in \Gamma_{B} \psi(x)$ for all $x \in X$. Then $g(x) \in H$ for all $x \in X$.

Let $\sigma: H \rightarrow \Re(M)$ be as in $\S 2$. Apply [3, Theorem 1.2] (that is, our Theorem 1.2 with metrizable domain) to the set-valued function $\sigma$, which is l.s.c. by Lemma 2.2 , to pick a continuous $h: H \rightarrow E$ such that $h(y) \in \Gamma_{E} \sigma(y)$ for all $y \in H$.

Define $f: X \rightarrow E$ by $f=g \circ h$. If $x \in X$, then $g(x) \in \Gamma_{E} \psi(x)$, so $\sigma(g(x))$ $\subset \psi(x) \subset \phi(x)$ by Lemma 2.1 (a), and hence

$$
f(x)=h(g(x)) \in \Gamma_{E} \sigma(g(x)) \subset \Gamma_{E} \phi(x) .
$$

That completes the proof.

\section{REFERENCES}

1. E. Michael, Continuous selections. I, Ann. of Math. 63 (1956), 361-382.

2. - A theorem on semi-continuous set-valued functions, Duke Math. J. 26 (1959), 647-652.

3. - Three mapping theorems, Proc. Amer. Math. Soc. 15 (1964), 410-415.

4. - A short proof of the Arens-Eells embedding theorem, Proc. Amer. Math. Soc. 15 (1964), 415-416.

UNIVERSITY OF WASHINGTON 